\title{
Supporting Information to: \\ Intramolecular Vibrational Force Fields for Linear Carbon Chains through an Adaptative Linear Scaling Scheme
}

\author{
Matteo Tommasini, Daniele Fazzi, Alberto Milani, Mirella Del Zoppo, Chiara Castiglioni, and Giuseppe Zerbi \\ Center for NanoEngineered MAterials and Surfaces (NEMAS), \\ Dipartimento di Chimica, Materiali e Ingegneria Chimica, G. Natta, \\ Politecnico di Milano, P.zza Leonardo da Vinci 32, I-20133 Milan, Italy
}

\section{ANALYSIS OF THE FORCE CONSTANTS MATRIX}

In Fig. 1 the structure of the stretching force constants matrix which defines the vibrational problem in the longitudinal space is illustrated. The elements $F_{i j}$ of the stretching matrix $\mathbf{F}$ can be represented by using a colour map. The plots of Fig. 1 give an overall view of the behaviour of the elements of $\mathbf{F}$ for a quite long polyyne, $\mathrm{H}_{-} \mathrm{C}_{18^{-}}$ $\mathrm{H}$, which extends for about $2 \mathrm{~nm}$ from hydrogen to hydrogen. The diagonal of $\mathbf{F}$ ( $\mathbf{f}_{0}$ vector) collects the diagonal force constants relative to the bond stretching coordinates along the chain. The co-diagonals $\left(\right.$ vectors $\mathbf{f}_{1}, \mathbf{f}_{2}, \ldots, \mathbf{f}_{s}$ ) collect the interactions between stretching coordinates along the chain which are respectively first nearest neighbours, second nearest neighbours, and so on [10]. Obviously the matrix F is symmetric. In Fig. 1 it is also reported a comparison between the force constant matrix obtained for $\mathrm{H}_{-} \mathrm{C}_{18}-\mathrm{H}$ at the Hartree-Fock level and with the hybrid DFT functional PBE1PBE with Gaussian03 code [2]. A look at the logarithmic plots of Fig. 1 reveals that the off-diagonal interactions $\mathbf{f}_{s}$ in the DFT calculation die off more slowly with respect to the interaction distance $s$ than in the Hartree-Fock calculation. As shown in the text, the effective range of interaction has profound effects on the calculation of the frequencies of the strongest Raman active vibration. Moreover, as the right panel of Fig. 1 shows, a regular and striking alternation of signs is found in the elements of $\mathbf{F}$. Neglecting force constants involving $\mathbf{C H}$ stretching coordinates (i.e. considering the sub matrix of the CC interactions, $F_{i j}, 2 \leq i, j \leq 18$ ), we can state that the elements of the vectors $\mathbf{f}_{2 s+1}$ (for $0 \leq s \leq 6$ ) are positive, while those of the vectors $\mathbf{f}_{2 s}$ (for $1 \leq s \leq 6$ ) are negative (the diagonal vector $\mathbf{f}_{0}$ is necessarily positive). The same behaviour has been found in the past for other $\pi$-conjugated systems and it has been fully rationalized in the frame of Hückel theory where expressions for CC stretching interaction force constants can be analytically obtained as a function of parameters describing the coupling between $\pi$ electrons and nuclear motions $[3,4]$.

A quantitative analysis of the decreasing behaviour of the off diagonal interactions belonging to $\mathbf{f}_{s}$ with respect to $s$, is done in Fig. 2 where a logarithmic plot is reported for the average value of $\mathbf{f}_{s}$ against the interaction distance $s$. Several chain lengths have been considered, ranging from 8 up to 20 carbon atoms. Interestingly, longer chains experience a slower decay of the average interaction force constants as a function of the interaction distance $s$. This behaviour can be related to the well known decrease of the HOMO-LUMO gap in poly-conjugated systems of increasing size and it is found in other $\pi$-systems affected by Kohn anomaly, such as graphite and carbon nanotubes [5, 6]. This effect is especially relevant in nanotubes [6] resulting in a slower decay of CC stretching interaction force constants in the case of metallic nanotubes with respect to the semiconducting ones. In a recent work where phonon dispersion of models of an infinite polyyne chain have been obtained [7], the long-range behaviour (slow decay) of the stretching interaction force constants has been pointed out and related to Kohn anomaly.

\section{EFFECT OF THE DFT FUNCTIONAL ON THE SIMULATED RAMAN SPECTRA}

We consider now more deeply how the choice of the functional affects the simulated Raman spectrum. We first focus on the longer polyyne, $\mathrm{H}_{-} \mathrm{C}_{20}-\mathrm{H}$, for which the interaction range is longer. In Fig. 3 we report the average value of the off-diagonal interactions $\left\langle\mathbf{f}_{s}\right\rangle$ as a function of the interaction distance $s$. Different widely used functionals have been considered, the hybrid B3LYP and PBE1PBE, the pure DFT functional PBEPBE (GGA) and SVWN (LDA). Hartree-Fock (RHF) data are also plotted for comparison. As the data reported in Fig. 3 clearly show, the inclusion of exact exchange affects the decay slope of the off-diagonal interactions which appears to be steeper. A hybrid DFT method provides shorter range vibrational interactions with respect to pure DFT. This fact of course is reflected in the simulated Raman spectra which are reported in the main text. A longer range of interactions, such as the one predicted by the PBEPBE functional, is associated with a significantly lower frequency of the $\alpha$ peak, which shifts downwards by roughly $100 \mathrm{~cm}^{-1}$ while passing from hybrid to pure DFT functional. The effect of the basis set is more limited, as we verified by comparing the PBEPBE calculations carried out with Dunning's correlation consistent basis set of double zeta quality (cc-pVDZ, not reported in this work) with respect to a triple zeta quality basis set 
(cc-pVTZ) and 6-311G**. Generally speaking, for a given DFT functional, the increase of the basis set produces an increase of the interaction range and hence a softening of the main Raman peak. This can be intuitively rationalized by considering that more extended basis sets are expected to describe a more polarizable atom, therefore providing dynamical interactions over a longer range. Fig. 4 of the main text reveals also that Hartree-Fock calculations predict only a minor downshift of the $F_{\mathcal{R}}$ parameter: consequently, the corresponding Raman spectra show only a minor dispersion of the $\alpha$ line. We therefore conclude that the inclusion of correlation effects is essential for predicting a reliable frequency dispersion. Fig. 1 of the main text shows that while hybrid functionals always overestimate the experimental frequencies, the PBEPBE functional gives a very good agreement for short molecules, but it yelds too low frequencies for increasing lengths, thus overestimating delocalization. On the other side, in the case of the hybrid PBE1PBE functional, the frequencies are always overestimated but the ratio between the Raman intensities of the $\alpha$ and $\beta$ Raman lines is well predicted, contrary to B3LYP or PBEPBE. We can thus conclude that none of the theoretical methods describes fully the experimentally observed trend [8]. Hence we need a new scaling procedure to obtain a better agreement with experiments. Due to the better agreement obtained for the $\alpha$ - $\beta$ intensities ratio, the PBE1PBE/cc-pVTZ results have been used to develop the adaptative linear scaling scheme.

\section{PHONONS OF A DIATOMIC LINEAR CHAIN AND NORMAL MODES OF FINITE CHAINS}

The treatment of the longitudinal phonons of a mono-atomic linear chain will be first considered following the discussion found in [9]. It can be shown that an oligomer made by $n$ units ( $n$ atoms) identical to that forming the infinite monoatomic chain gives rise to $n$ longitudinal normal vibrations whose frequencies exactly fit the dispersion branch of the infinite chain (only one LA branch). The vibrational frequencies of the oligomers are found along the dispersion curve of the $1 \mathrm{D}$ crystal exactly for these values of the wavevector [9]:

$$
\begin{aligned}
& q=\frac{\pi}{a} \frac{j}{n} ; \quad(j=0, n-1) \quad \text { chain with free ends } \\
& q=\frac{\pi}{a} \frac{j}{n+1} ; \quad(j=1, n) \quad \text { chain with fixed ends }
\end{aligned}
$$

According to Eqs. $(1,2)$ it is clear that the modelling of the ends of the oligomer chain is critical for the choice of the appropriate $q$ wave-vectors, especially in the case of very short chains. Real chains with end groups chemically different from the atoms in the backbone are usually treated according to the fixed end model. This choice mimics the fact that vibrational diplacements of the end atoms are generally de-coupled from vibrational motions of the backbone. In the case under study (hydrogen capped polyynes), this is directly proven by the appearance of two high frequencies (close to $3268 \mathrm{~cm}^{-1}$ ) assigned to gerade and ungerade localized $\mathrm{CH}$ stretching normal modes. While Eq. (2) holds in the case of monoatomic chains, it is relatively simple to derive a similar relationship for a dimerized chain, making use of the concept of "zone folding". Dispersion relations for the diatomic chain can be regarded as obtained by doubling the crystal unit cell (thus halving the first BZ) and folding the LA phonon branch at $\pi / a$ into the $\Gamma$ point thus opening of a gap at $q=\pi /(2 a)$. In this way a LO branch is obtained. It can be realized that this procedure provides a new form of Eq. (2), where wave-vectors are now referred to the dimerized crystal with $c=2 a$ cell parameter:

$$
q=\frac{\pi}{c} \frac{2 j}{n+1}=\frac{\pi}{c} \frac{j}{N+1 / 2} ; \quad(j=1, N)
$$

$N$ being the number of triple bonds $(N=n / 2)$ in the oligomer chain.

Following Eq. (3) it has been possible to put all the calculated $\mathrm{CC}$ stretching frequencies of an oligoyne $(\mathrm{N}=7)$ onto the phonon curves previously obtained for the relative infinite model. The frequencies of the oligomer fit very well the phonon branches. Some minor discrepancies are due to the following facts: (i) real ends cannot be treated with the simple "fixed ends" model; (ii) due to confinement effects interaction force constants relative to different units along the oligomer chain are slightly different, while the $\mathbf{F}$ matrix of the infinite chain has been built by considering just the force constants relative to the central unit of the oligoyne with $\mathrm{N}=7$ triple bonds.

Moreover, according to the above treatment, it is now possible to assign a suitable phonon wave-vector to normal modes associated to $\alpha$ and $\beta$ Raman lines of polyynes; they are located on the LO branch and obey to the following relationships:

- $\alpha$ lines:

$$
q=\frac{\pi}{c} \frac{1}{N+1 / 2}
$$


- $\beta$ lines:

$$
\begin{array}{rlrl}
q=\frac{\pi}{c} \frac{N}{N+1 / 2} ; & & (N \text { odd }) \\
q=\frac{\pi}{c} \frac{N-1}{N+1 / 2} ; & (N \quad \text { even })
\end{array}
$$

In other words: for a given polyyne the $\alpha$ line corresponds to the allowed wave-vector (for the finite chain) closest to $\Gamma$ point $(q \approx 0)$ and the $\beta$ line to the allowed wave-vector closest to $q=\pi / c$ and corresponding to a Raman active normal mode. Notice moreover that any given oligoyne has a different force field [7]: for this reason the ideal phonon dispersion curve to which its frequencies are associated are different.

[1] Yang, S.; Kertesz, M.; Zólyomi, V.; Kürti, J. J. Phys. Chem. A 2007, 111, 2434.

[2] Gaussian 03, Revision C.02, Frisch, M. J.; Trucks, G. W.; Schlegel, H. B.; Scuseria, G. E.; Robb, M. A.; Cheeseman, J. R.; Montgomery, Jr., J. A.; Vreven, T.; Kudin, K. N.; Burant, J. C.; Millam, J. M.; Iyengar, S. S.; Tomasi, J.; Barone, V.; Mennucci, B.; Cossi, M.; Scalmani, G.; Rega, N.; Petersson, G. A.; Nakatsuji, H.; Hada, M.; Ehara, M.; Toyota, K.; Fukuda, R.; Hasegawa, J.; Ishida, M.; Nakajima, T.; Honda, Y.; Kitao, O.; Nakai, H.; Klene, M.; Li, X.; Knox, J. E.; Hratchian, H. P.; Cross, J. B.; Bakken, V.; Adamo, C.; Jaramillo, J.; Gomperts, R.; Stratmann, R. E.; Yazyev, O.; Austin, A. J.; Cammi, R.; Pomelli, C.; Ochterski, J. W.; Ayala, P. Y.; Morokuma, K.; Voth, G. A.; Salvador, P.; Dannenberg, J. J.; Zakrzewski, V. G.; Dapprich, S.; Daniels, A. D.; Strain, M. C.; Farkas, O.; Malick, D. K.; Rabuck, A. D.; Raghavachari, K.; Foresman, J. B.; Ortiz, J. V.; Cui, Q.; Baboul, A. G.; Clifford, S.; Cioslowski, J.; Stefanov, B. B.; Liu, G.; Liashenko, A.; Piskorz, P.; Komaromi, I.; Martin, R. L.; Fox, D. J.; Keith, T.; Al-Laham, M. A.; Peng, C. Y.; Nanayakkara, A.; Challacombe, M.; Gill, P. M. W.; Johnson, B.; Chen, W.; Wong, M. W.; Gonzalez, C.; and Pople, J. A.; Gaussian, Inc., Wallingford CT, 2004.

[3] (a) Coulson, C. A.; Longuet-Higgins, H. C. Proc. R. Soc. London A 1947, 191, 39; (b) Coulson, C. A.; Longuet-Higgins, H. C. Proc. R. Soc. London A 1948, 193, 447; (c) Coulson, C. A.; Longuet-Higgins, H. C. Proc. R. Soc. London A 1948, $193,456$.

[4] (a) Gussoni, M.; Castiglioni, C.; Zerbi, G. in Spectroscopy of advanced materials, Clark, J. H.; Hester, R. E. eds., Wiley and Sons, 1991; (b) Castiglioni, C.; Tommasini, M.; Zerbi, G. Phil. Trans. Roy. Soc. A 2004, 362, 2425.

[5] Piscanec, S.; Lazzeri, M.; Mauri, F.; Ferrari, A. C.; Robertson, J. Phys. Rev. Lett. 2004, $93,185503$.

[6] Di Donato, E.; Tommasini, M.; Castiglioni, C.; Zerbi, G. Phys. Rev. B 2006, 74, 184306.

[7] Milani, A.; Tommasini, M.; Del Zoppo, M.; Castiglioni, C.; Zerbi, G. Phys. Rev.B 2006, 74, 153418.

[8] Tabata, H.; Fujii, M.; Hayashi, S.; Doi, T.; Wakabayashi, T. Carbon 2006 44, 3168.

[9] Zbinden, R. Infrared Spectroscopy of High Polymers, Academic Press: New York, 1964.

[10] The $s$ index corresponds to the difference $|i-j|$ which measures the interaction distance between the $i$-th and $j$-the CC bond, according to the notation introduced in [1]. 
(a) RHF

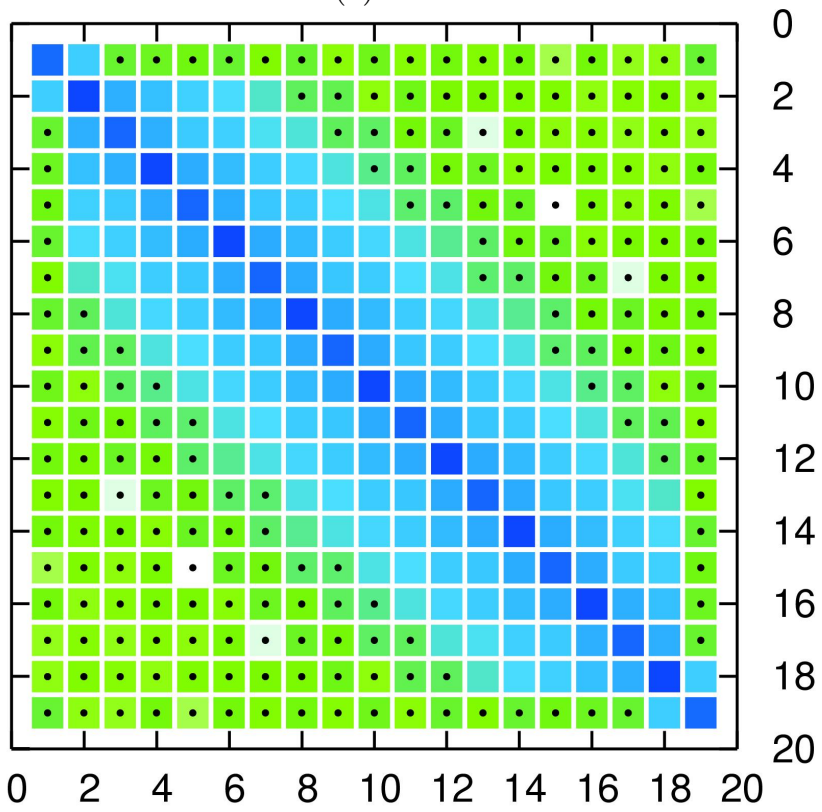

(b) PBE1PBE

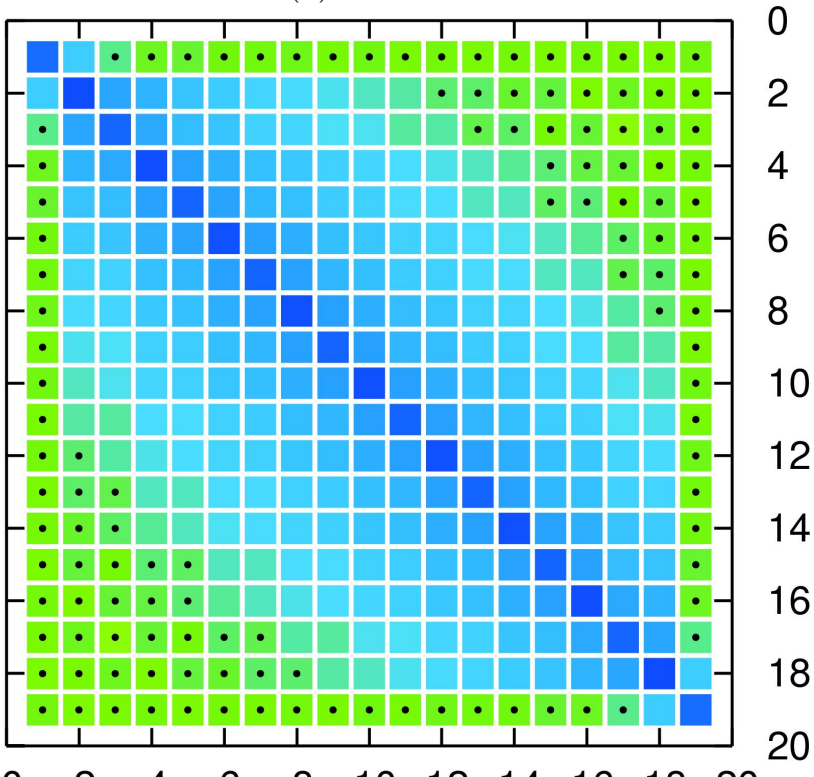

$\begin{array}{lllllllllll}0 & 2 & 4 & 6 & 8 & 10 & 12 & 14 & 16 & 18 & 20\end{array}$
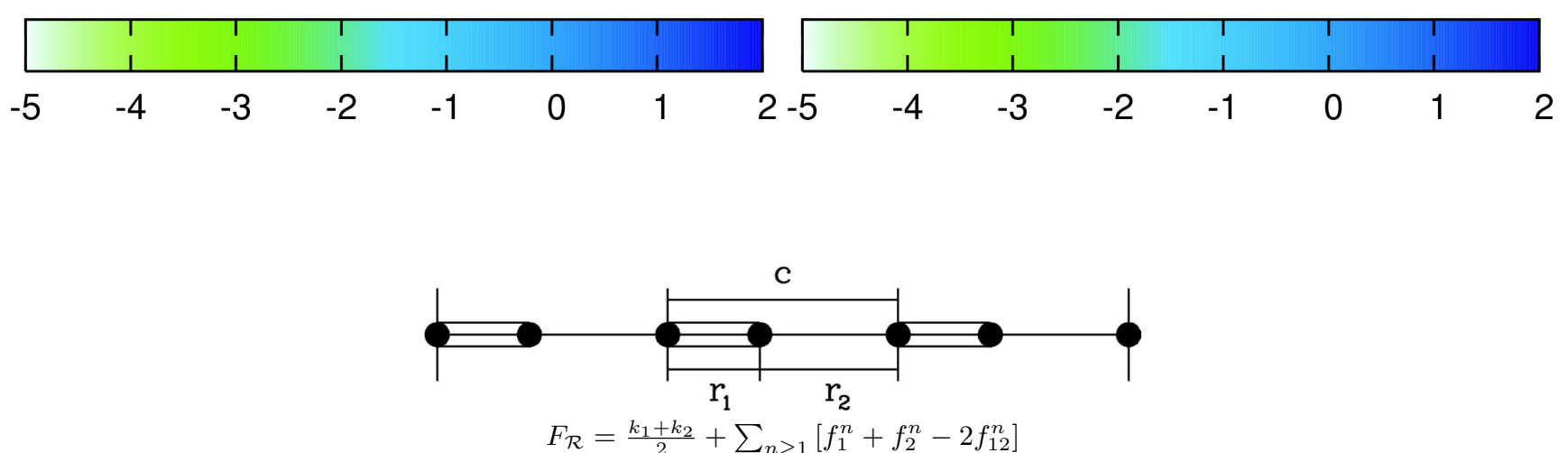

\begin{tabular}{|c|c|c|c|c|c|c|c|}
\hline$k_{1}$ & $f_{12}^{1}$ & $f_{1}^{1}$ & $f_{12}^{2}$ & $f_{1}^{2}$ & $f_{12}^{3}$ & $f_{1}^{3}$ & $\ldots$ \\
\hline & $k_{2}$ & $f_{12}^{1}$ & $f_{2}^{1}$ & $f_{12}^{2}$ & $f_{2}^{2}$ & $f_{12}^{3}$ & $f_{2}^{3}$ \\
\hline & & $k_{1}$ & $f_{12}^{1}$ & $f_{1}^{1}$ & $f_{12}^{2}$ & $f_{1}^{2}$ & $f_{12}^{3}$ \\
\hline & & & $k_{2}$ & $f_{12}^{1}$ & $f_{2}^{1}$ & $f_{12}^{2}$ & $f_{2}^{2}$ \\
\hline & & & & $k_{1}$ & $f_{12}^{1}$ & $f_{1}^{1}$ & $f_{12}^{2}$ \\
\hline & & & & & $k_{2}$ & $f_{12}^{1}$ & $f_{2}^{1}$ \\
\hline & & & & & & $k_{1}$ & $f_{12}^{1}$ \\
\hline & & & & & & & $k_{2}$ \\
\hline
\end{tabular}

FIG. 1: Top (a, b): logarithmic colour map of the force constant matrix $\mathbf{F}$ of the polyyne $\mathrm{H}_{-} \mathrm{C}_{18}-\mathrm{H}$ restricted to the bond stretching internal coordinates (the colour map is relative to $\log _{10}\left|F_{i j}\right| / k$, where $k$ equals to the adopted unit, namely 1 mdyne/A). The CC stretching coordinates run from index 2 to index 18 and are arranged in an ordered sequence extending from one end of the linear chain to the other. The coordinates number 1 and 20 are $\mathrm{CH}$ stretchings. The data reported here have been obtained with a restricted Hartree-Fock calculation (a) and a PBE1PBE DFT calculation (b). In both cases the basis set is cc-pVTZ. Force constants lower than $10^{-2}$ mdyne $/ \AA$ are marked with a black spot to more clearly show the longer range of interaction of PBE1PBE with respect to Hartree-Fock. Bottom: symbolic representation of a limited part of the force constant matrix $\mathbf{F}$ of an infinite periodic linear carbon chain (for clarity just the upper triangular part of the symmetric matrix is shown). Red matrix elements are positive, in agreement to previous studies [4]. A sketch of the unit cell of the infinite chain with alternated bonds is also reported. The shaded matrix elements are those used to compute $F_{\mathcal{R}}$ according to the equation reported. 


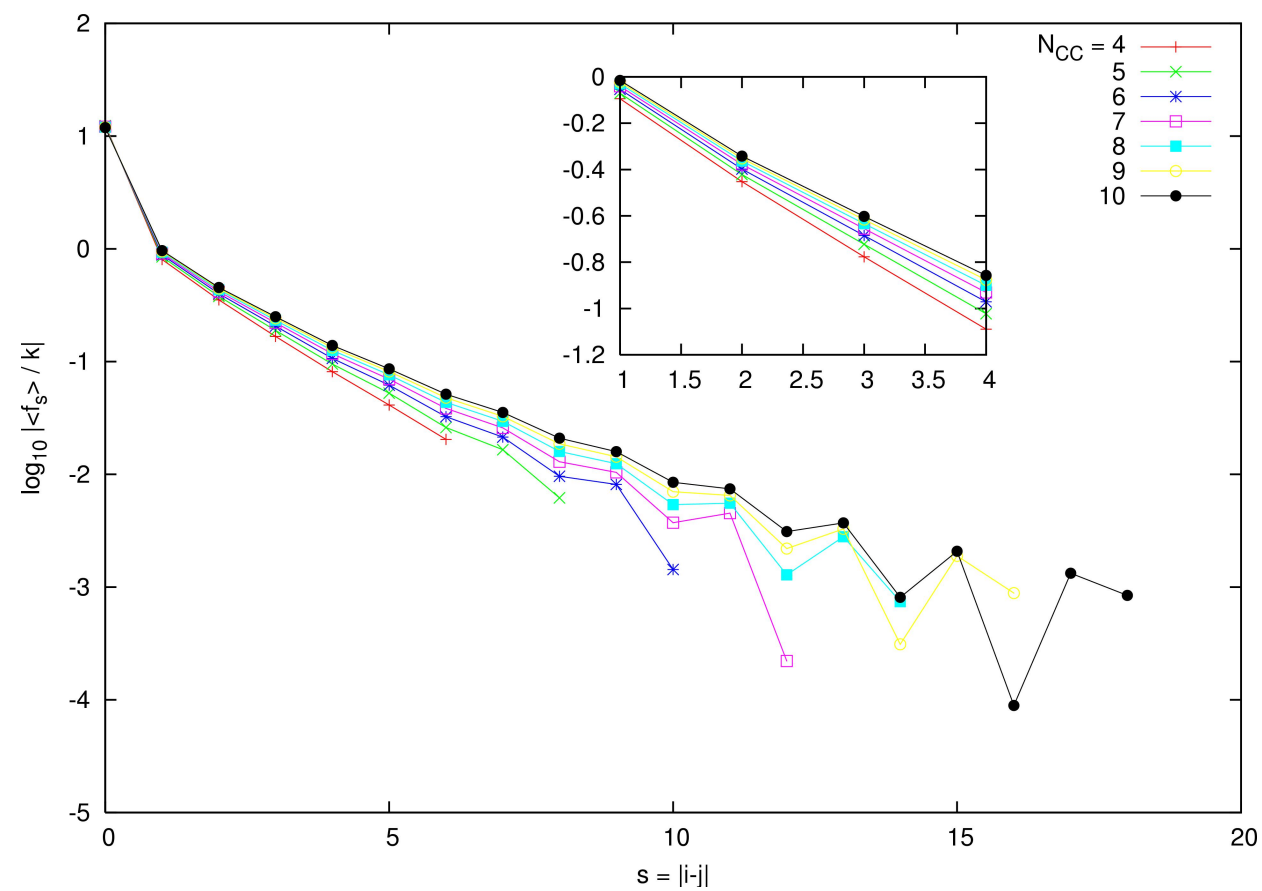

FIG. 2: Logarithmic plot of the decay of the average force constant $\left\langle\mathbf{f}_{s}\right\rangle$ with respect to the distance of interaction $s=|i-j|$ between the $i$-th and $j$-th CC bond $(k=1$ mdyne $/ \AA)$. The data reported here have been obtained with DFT calculations at PBE1PBE/cc-pVTZ level carried out for polyynes of increasing lengths, $\mathrm{H}-\mathrm{C}_{2 N}-\mathrm{H}(4 \leq N \leq 10)$. The inset plot shows a zoom of the data for $1 \leq s \leq 4$. The increase of the range of interaction with increasing chain length is evident.

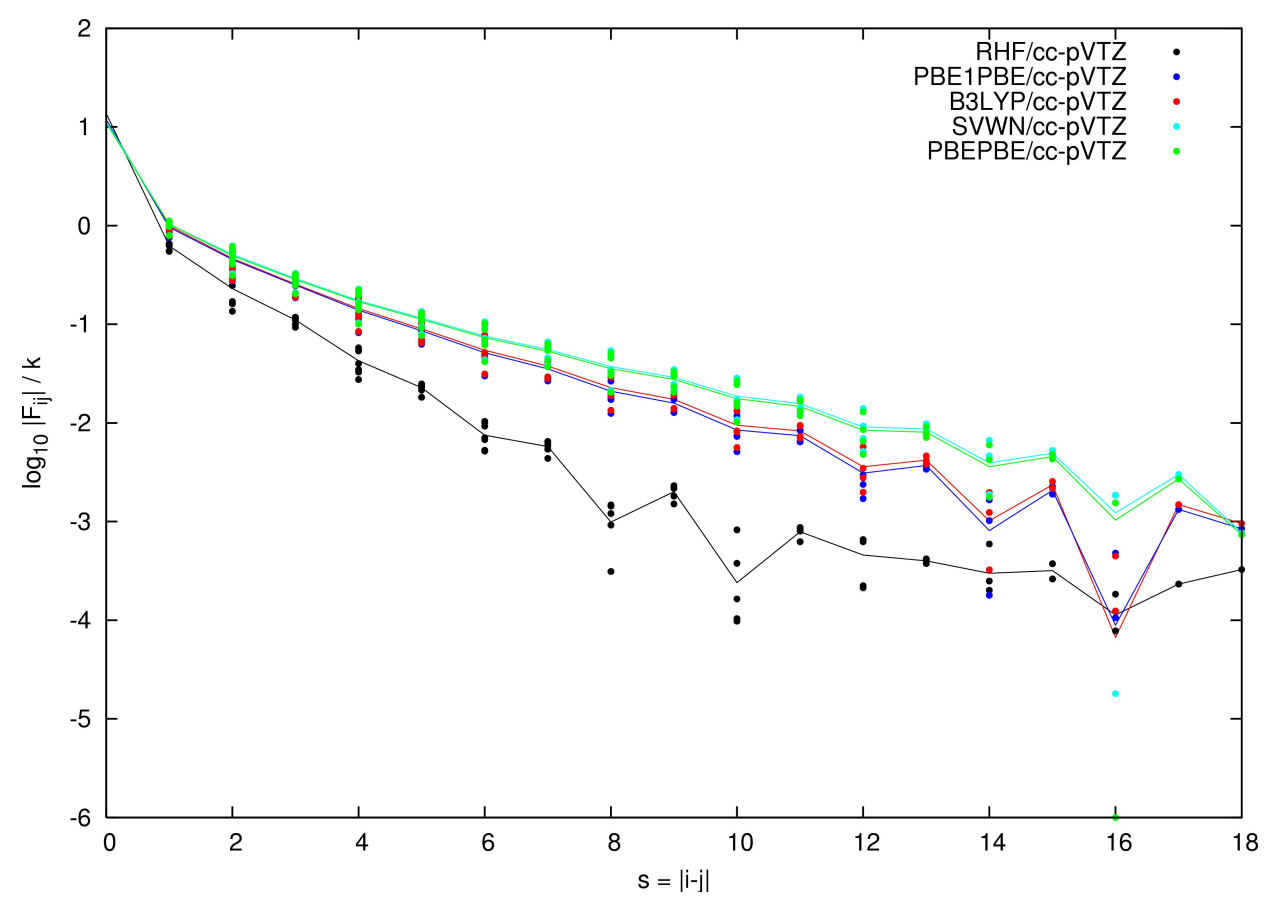

FIG. 3: Double logarithmic plot of the decay of the average force constant $\left\langle\mathbf{f}_{s}\right\rangle$ with respect to the distance of interaction $s$; the logarithm of the single matrix elements $\left|F_{i j}\right| / k$ is also shown as a function of $s=|i-j|,(k=1$ mdyne/A). The data reported here have been obtained with hybrid DFT functionals (B3LYP, PBE1PBE) and pure DFT functionals [SVWN (LDA) and PBEPBE (GGA)] for a long polyyne of fixed length, $\mathrm{H}-\mathrm{C}_{20}-\mathrm{H}$. RHF values are plotted for a comparison. All the calculations have been carried out by using cc-pVTZ basis set. 

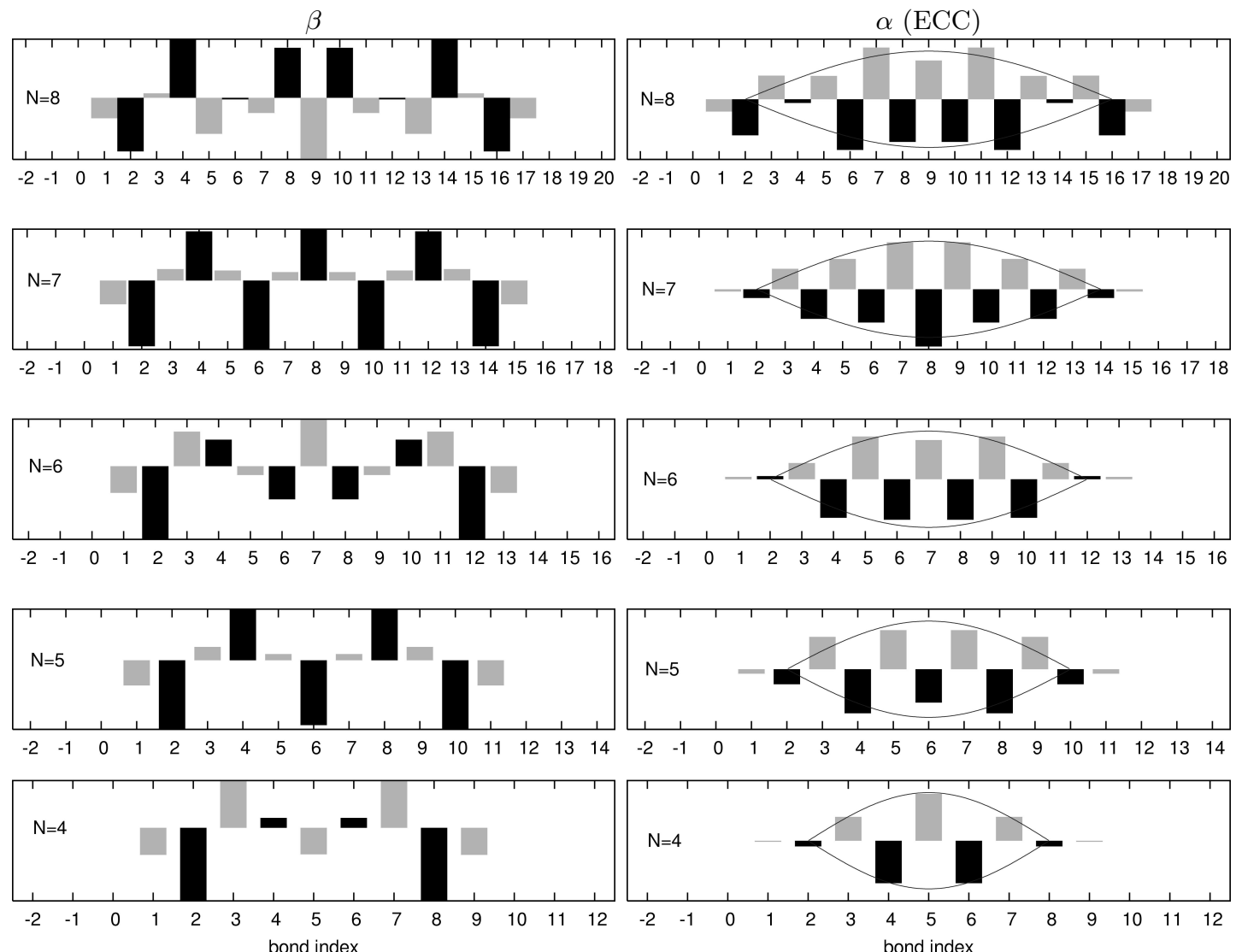

FIG. 4: Nuclear displacements associated to the $\alpha$ and $\beta$ lines obtained with PBE1PBE/cc-pVTZ calculations scaled with the linear adaptative procedure (see text). 